\title{
OPEN Assessment of copy number in protooncogenes are predictive of poor survival in advanced gastric cancer
}

\author{
Meihui $\mathrm{Li}^{1,2,3}$, Younghoon $\mathrm{Kim}^{1,2,3}$, Tae-Shin $\mathrm{Kim}^{2}$, Nam-Yun $\mathrm{Cho}^{1,2}$, Jeong Mo Bae ${ }^{1,2}$, \\ Woo Ho Kim ${ }^{1}$ \& Gyeong Hoon $\mathrm{Kang}^{1,2 \varpi}$
}

The copy number (CN) gain of protooncogenes is a frequent finding in gastric carcinoma (GC), but its prognostic implication remains elusive. The study aimed to characterize the clinicopathological features, including prognosis, of GCs with copy number gains in multiple protooncogenes. Three hundred thirty-three patients with advanced GC were analyzed for their gene ratios in EGFR, GATA6, IGF2, and SETDB1 using droplet dPCR (ddPCR) for an accurate assessment of CN changes in target genes. The number of GC patients with 3 or more genes with $\mathrm{CN}$ gain was 16 (4.8\%). Compared with the GCs with 2 or less genes with CN gain, the GCs with 3 or more CN gains displayed more frequent venous invasion, a lower density of tumor-infiltrating lymphocytes, and lower methylation levels of L1 or SAT-alpha. Microsatellite instability-high tumors or Epstein-Barr virus-positive tumors were not found in the GCs with 3 or more genes with CN gain. Patients of this groups also showed the worst clinical outcomes for both overall survival and recurrence-free survival, which was persistent in the multivariate survival analyses. Our findings suggest that the ddPCR-based detection of multiple CN gain of protooncogenes might help to identify a subset of patients with poor prognosis.

Gastric carcinoma (GC) is one of the most common malignancies in Eastern Asia and one of the leading causes of cancer-related deaths. TNM cancer staging provides prognostic information, but clinical outcomes vary among patients with GC of the same cancer stage. For patients with GC of the same cancer stage, further prognostic information could be gained from biomarkers including pathological parameters, such as lymphovascular invasion, perineural invasion, and tumor-infiltrating lymphocytes (TILs). Molecular markers might provide information about the prognostic features of the tumor. The Cancer Genome Atlas (TCGA) project has defined four subtypes of GC, including GCs with microsatellite instability (MSI), Epstein-Barr virus (EBV), genomic stability (GS), and chromosomal instability (CIN), which have been associated with different prognoses ${ }^{1,2}$. The EBV subtype was associated with the best prognosis, while the GS subtype was associated with the worst prognosis. Although the CIN subtype fell in between the above two subtypes, it demonstrated the greatest survival benefit from adjuvant chemotherapy ${ }^{1}$, which indicates that the molecular subtyping of GCs might provide prognostic and predictive value.

CIN consists of numerical and/or structural aberrations in chromosomes. Numerical abnormality refers to the gain or loss of whole chromosomes, whereas structural abnormalities include the amplification, loss, translocation, and inversion of chromosomal regions of various sizes ranging from a single gene to an arm. Through the TCGA project, many genes have been found to be amplified or undergo copy number gain, including EGFR, FGFR1, GATA6, HER2 (ERBB2), IGF2, MYC, and SETDB1 in $\mathrm{GCs}^{2}$. Although copy number gains of these genes are expected to occur mainly in the CIN subtype of GC, the prognostic significance of the copy number gains of these genes has not yet been clarified.

Digital polymerase chain reaction ( $\mathrm{dPCR}$ ) is a method that provides quantitative information about copy number changes in probed genes without the need for standard curves. DNA samples obtained from formalinfixed archival tissues contain inhibitors for PCR and formalin-induced interstrand crosslinking, which can result in errors in the analysis of copy number variation by quantitative PCR (qPCR). However, dPCR can provide

${ }^{1}$ Department of Pathology, Cancer Research Institute, Seoul National University College of Medicine, 103 Daehak-ro, Jongno-gu, Seoul 03080, Korea. '2Laboratory of Epigenetics, Cancer Research Institute, Seoul National University College of Medicine, Seoul, Korea. ${ }^{3}$ These authors contributed equally: Meihui Li and Younghoon Kim. ${ }^{凶}$ email: ghkang@snu.ac.kr 
more accurate results because it does not use the comparison of PCR rates relied on by qPCR but instead uses the determination of whether amplification above a threshold has occurred. In the present study, we aimed to elucidate whether copy number changes in seven genes (EGFR, FGFR1, GATA6, HER2, IGF2, MYC, and SETDB1) are related to the survival of patients with advanced GC and might serve to detect a subset of GC cases with poor prognosis. The genes included in this study belong to those which are most frequently amplified in TCGA. We used droplet dPCR (ddPCR) to evaluate the copy number changes of the seven genes in formalin-fixed, paraffin embedded tissue samples of advanced GC.

\section{Materials and methods}

Samples. A total of 333 formalin-fixed, paraffin-embedded GC tissues were retrieved from the surgical files of the Department of Pathology, Seoul National University Hospital, Seoul, Korea. The patients underwent surgery and extended lymph node dissection (D2) for advanced GC (T2-T4) from 2007 to 2008. Patients were included in the study according to the following criteria: age at diagnosis $>18$ years, advanced GC, adenocarcinoma histology, and availability of formalin-fixed paraffin-embedded (FFPE) cancer tissues. The exclusion criteria included patients who had a history of other primary malignancies (except for papillary thyroid cancer) within 5 years or received chemotherapy before surgical resection. Clinical and histological information was obtained from electronic medical records, including Lauren histology, tumor subsite within the stomach, lymphatic embolus, venous invasion status, perineural invasion status, and tumor-node-metastasis (TNM) stage (American Joint Committee on Cancer, 7th edition). The patients were previously analyzed for their EBV infection, MSI, and tumoral L1 and SAT-alpha methylation statuses ${ }^{3,4}$. A tissue microarray was constructed from the tumor center and immunostained against CD3 and CD8. TILs were counted in the CD3 and CD8immunostained cores $(0.2 \mathrm{~cm}$ in diameter), and CD3 and CD8 TIL densities were determined in a previous study $^{5}$. For the ddPCR assay, on glass slides, we marked the tumor areas with the highest tumor purity and the most representative histology of the case under the microscope and then manually dissected the corresponding tumor areas on three to five unstained serial sections $(10 \mu \mathrm{m}$-thick). The dissected tissues were subjected to DNA extraction and purification using a QIAamp DNA FFPE Tissue kit (Qiagen Gmbh, Hilden, Germany). Purified DNA was quantified with a Qubit 2.0 fluorometer (Thermo Scientific, Wilmington, DE). As control DNA, normal genomic DNA was extracted from the nonneoplastic gastric mucosa of GC patients and from the white blood cells of healthy volunteers using the QIAamp DNA Mini Kit (Qiagen). This study was approved by the Institutional Review Board of Seoul National University Hospital, which waived the requirements to obtain informed patient consent (approval no. H-1312-051-542). All procedures performed in studies involving human participants were in accordance with the ethical standards of the institutional and/or national research committee and with the 1964 Helsinki declaration and its later amendments or comparable ethical standards.

Design of primers and probes. The primers and probe sequences of one protooncogene (EGFR) and reference gene (Ribonuclease P RNA component H1 (RPPH1)) are listed in Supplementary Table $1^{6}$ and were synthesized by Integrated DNA Technologies (Coralville, IA, USA). The primers and probes of the other six genes, including MYC, HER2 (ERBB2), FGFR1, GATA6, IGF2, and SETDB1, were purchased from Life Technologies (Carlsbad, CA, USA). RPPH1 was used as a reference locus because it is a highly conserved region that is present at 1 copy per haploid genome.

Droplet digital PCR. Both the target gene and RPPH1 loci were amplified simultaneously in duplex PCR. PCR mixtures were made with ddPCR Supermix for Probes (Bio-Rad, Hercules, CA) according to the manufacturer's protocol. Each $20 \mu \mathrm{l}$ reaction mixture contained $100 \mathrm{ng}$ of DNA, $900 \mathrm{nM}$ of forward and reverse primers, and $250 \mathrm{nM}$ of probes. We omitted the restriction enzyme digestion of DNA because formalin-fixed DNA tends to be highly fragmented. dPCR was performed on the QX200 ddPCR system (Bio-Rad). In brief, $20 \mu \mathrm{l}$ of the PCR mixture was partitioned into an emulsion of approximately 20,000 uniformly sized droplets via a QX200 droplet generator. The droplets were transferred to a 96-well PCR plate, heat-sealed, and placed in a conventional thermal cycler (T100, Bio-Rad). The thermal cycling conditions were $95^{\circ} \mathrm{C}$ for $10 \mathrm{~min} ; 40$ cycles of $94{ }^{\circ} \mathrm{C}$ for $30 \mathrm{~s}, 57^{\circ} \mathrm{C}$ for $50 \mathrm{~s}$ and $72{ }^{\circ} \mathrm{C}$ for $30 \mathrm{~s} ; 98^{\circ} \mathrm{C}$ for $10 \mathrm{~min}$; and a $12{ }^{\circ} \mathrm{C}$ hold. After PCR, the plate was loaded on a QX200 droplet reader for the automatic detection of the fluorescence in each well. Analysis of the ddPCR data was performed with QuantaSoft software (Bio-Rad).

Pyrosequencing methylation assay of L1 and SAT-alpha. After bisulfite modification of the extracted DNA, the modified DNA was subjected to pyrosequencing methylation assays of L1 and SAT-alpha. The detailed procedures and determination of methylation levels were described in a previous study ${ }^{4}$.

Quantitative real-time RT-PCR (qRT-PCR). Total RNA was extracted from cells using RNeasy Plus Mini Kit (QIAGEN). First-strand cDNA was synthesized with LeGene Premium Express 1st Strand cDNA Synthesis System (LeGene Biosciences, San Diego, CA, USA) and either stored at $-20^{\circ} \mathrm{C}$ or used immediately. Quantitative RT-PCR (RT-qPCR) reaction and analysis were performed using Bio-Rad iQ5 System (BioRad, Hercules, CA, USA). SYBR Green PCR Master Mix (Thermo Fisher Scientific, Waltham, MA, USA) was used for SYBR Green-based RT-qPCR according to the manufacturer's protocol. Primers used in RT-qPCR reactions were purchased from the Integrated DNA Technologies (Coralville, IA, USA) and listed in Supplementary Table 2. Each qPCR analysis was done in triplicate, and the average of the triplicate values represents every single result of the qPCR analysis. Fold changes in gene expression of test and control samples were determined by using the $2^{-\Delta \Delta C t}$ method. Relative quantity (RQ) is $2^{-\Delta \Delta \mathrm{Ct}}$, and copy number variation $(\mathrm{CNV})$ is $2 \times \mathrm{RQ}$. 
Cell culture. Cell lines SNU-1, SNU-5, SNU-16, SNU-216, SNU-484, SNU-601, SNU-620, SNU-638, SNU668, SNU-719, MKN-28, MKN-45, and MKN74 were cultured in RPMI-1640 (Welgene Co., Daegu, Korea) supplemented with 10\% heat-inactivated FBS (Fetal bovine serum) (Gibco, Grand Island, NY, USA), 100 U/ml penicillin, and $100 \mu \mathrm{g} / \mathrm{ml}$ streptomycin (Gibco). FU-97 cell lines were cultured in Dulbecco's modified Eagle's medium (DMEM) supplemented with 10\% heat-inactivated FBS (Fetal bovine serum) (Gibco, Grand Island, NY, USA), $100 \mathrm{U} / \mathrm{ml}$ penicillin, $100 \mu \mathrm{g} / \mathrm{ml}$ streptomycin (Gibco), and $10 \mathrm{mg} / \mathrm{L}$ Insulin (Sigma-Aldrich, St. Louis, $\mathrm{MO}, \mathrm{USA})$. All cells were grown in an incubator with $5 \% \mathrm{CO}_{2}$ at $37^{\circ} \mathrm{C}$.

Statistical analysis. Statistical analyses were performed using SPSS software for Windows, version 25.0 (IBM, Armonk, NY, USA). Two-sided $P$-values of less than 0.05 were considered statistically significant. To identify whether the gene ratios were normally distributed in GC tissue samples, normalization tests were performed for the gene ratios, which revealed that the gene ratios were not normally distributed. The mean values of the gene ratios across two groups or across three or more groups were compared using both Student's t-test and the Mann-Whitney test and both ANOVA and the Kruskal-Wallis test, respectively. The clinical outcome data were last updated in December 2019. Of the included 333 patients, 14 patients were lost to follow-up. Recurrencefree survival (RFS) was measured from the date of surgery for advanced GC to the date of the first documented recurrence or the date of death from any cause, whichever occurred first. Overall survival (OS) was calculated from the date of resection to the date of death from any cause or the last clinical follow-up time. Survival curves were assessed using the Kaplan-Meier method and the log rank test. Multivariate comparisons of survival rates were performed with the Cox proportional hazards regression model, and baseline characteristics were adjusted using a backward stepwise regression model including covariates of prognostic value.

\section{Results}

A total of 333 advanced GC patients were analyzed for their gene ratios using ddPCR. The demographic findings are summarized in Supplementary Table 3. The mean age of the patients was 60.8 years, with a median age of 61 years (ranging from 29 to 86 years). The male to female ratio was 223:110. The cancer stage was IB in 30 patients, II in 108 patients, and III in 195 patients. Regarding the tumor subsite within the stomach, 92 cases involved the upper one-third (high body and cardia), while the others did not involve the upper one-third. Lauren's histology was intestinal type in 125 cases, diffuse type in 161 cases, mixed type in 43 cases, and unclassified in 4 cases. The molecular subtype was the MSI subtype in 42 cases, the Epstein-Barr virus subtype in 26 cases, and the non-MSI/non-EBV subtype in 265 cases.

Gene ratios in gastric cancer tissues and their normality tests. To identify whether the gene ratio ranged from approximately 1 in normal cells, we analyzed the statuses of the seven genes in the peripheral blood leukocytes of normal volunteer subjects $(n=20)$. The average values of the probed gene ratio ranged from 0.89 to 1.30 with standard deviation values less than 0.196 . However, for GC tissue samples, the average values of the seven gene ratios ranged from 0.84 to 43.5 (Supplementary Table 4). To identify whether the gene ratios were normally distributed in the GC tissue samples, a normalization test was performed using the Shapiro-Wilk test, which showed that the gene ratios were non-normally distributed.

Gene ratios and survival. For the survival analysis, the GC patients were grouped into 10 equal-sized subsets (i.e., each group has approximately the same number of patients), from subset 1 to subset 10, according to the increasing order of the gene ratios of the individual genes. With the Kaplan-Meier log rank test, EGFR, FGFR1, GATA6, IGF2, and SETDB1 showed lower survival in subset 10 than in the other subsets (Supplementary Fig. 1 \& 2). When the patients were divided into subset 10 and the other subsets, EGFR, FGFR1, GATA6, IGF2, and SETDB1 exhibited significant differences in survival time between subset 10 and the other subsets in the Kaplan-Meier log rank test (Supplementary Fig. $3 \& 4$ ). The clinicopathological and molecular features that were found to be statistically significant in univariate survival analysis included tumor subsite, Lauren classification, $\mathrm{T}$ stage, $\mathrm{N}$ stage, $\mathrm{M}$ stage, venous invasion, lymphatic embolus, and perineural invasion. When the individual genes were included in multivariate survival analysis with clinicopathological factors that were found to be significantly associated with survival, the EGFR and IGF2 gene ratios were independent prognostic parameters associated with poor prognosis in terms of both OS and RFS (Table 1). The GATA6 gene ratio was found to be a significant risk factor in the multivariate analysis of OS only, and the SETDB1 gene ratio was found to be a significant risk factor in the multivariate analysis of RFS only.

To evaluate the additive effect of copy number gains in four genes (EGFR, GATA6, IGF2, and SETDB1) on prognostication power, a tumor was scored " 1 " or " 0 " when the specific gene ratio belonged to subset 10 or the other subsets, respectively. The sum of scores for the four genes ranged from 0 to 4 in each tumor. Although the survival curves of the sum scores were significantly different for OS and RFS (Kaplan-Meier log rank test), the survival curves of sum scores 1 and 2 were similar, and those of sum scores 3 and 4 were similar (Fig. 1a,b). Thus, the GC patients were classified into 3 subsets, including a subset with sum score 0 , a subset with sum score 1 or 2 , and a subset with sum score 3 or 4 (Fig. 1c,d). The sum scores of 3 and 4 were also independent prognostic factors of $\mathrm{OS}(\mathrm{HR}=3.805,95 \% \mathrm{CI}=2.014-7.188, P<0.001)$ and $\mathrm{RFS}(\mathrm{HR}=3.709,95 \% \mathrm{CI}=1.953-7.042, P<0.001)$ in GC patients regardless of tumor subsite, Lauren histology, venous invasion, lymphatic invasion, perineural invasion, and $\mathrm{T}, \mathrm{N}$, and $\mathrm{M}$ categories (Table 2).

To identify whether CNV determined by ddPCR was correlated with expression levels of mRNA in four genes, we measured mRNA expression levels of four genes in 14 gastric cancer cell lines, using RT-qPCR, which were analyzed for their CNV in four genes using ddPCR. Four genes showed significant correlations between RT-qPCR and ddPCR values (Fig. 2). 


\begin{tabular}{|c|c|c|c|c|c|c|}
\hline & \multicolumn{3}{|c|}{ Univariate analysis } & \multicolumn{3}{|c|}{ Multivariate analysis $^{\mathrm{a}}$} \\
\hline & HR & 95\% C.I & $P$-value & HR & 95\% C.I & $P$-value \\
\hline \multicolumn{7}{|l|}{ Overall survival } \\
\hline EGFR (subset 10 vs. subsets 1-9) & 1.895 & $1.202-2.986$ & 0.006 & 1.960 & $1.224-3.140$ & 0.005 \\
\hline FGFR1 (subset 10 vs. subsets 1-9) & 1.626 & $0.991-2.666$ & 0.054 & 1.153 & $0.665-2.000$ & 0.613 \\
\hline GATA6 (subset 10 vs. subsets $1-9$ ) & 2.104 & $1.335-3.316$ & 0.001 & 1.927 & $1.182-3.140$ & 0.008 \\
\hline HER2 (subset 10 vs. subsets $1-9$ ) & 0.804 & $0.445-1.452$ & 0.469 & 1.025 & $0.546-1.922$ & 0.939 \\
\hline IGF2 (subset 10 vs. subsets 1-9) & 2.328 & $1.487-3.644$ & 0.000 & 2.770 & $1.730-4.435$ & $<0.001$ \\
\hline MYC (subset 10 vs. subsets 1-9) & 1.161 & $0.680-1.982$ & 0.586 & 0.999 & $0.569-1.754$ & 0.998 \\
\hline SETDB1 (subset 10 vs. subsets 1-9) & 1.564 & $1.011-2.418$ & 0.044 & 1.482 & $0.954-2.303$ & 0.080 \\
\hline \multicolumn{7}{|l|}{ Recurrence-free survival } \\
\hline EGFR (subset 10 vs. subsets $1-9$ ) & 1.745 & $1.109-2.747$ & 0.016 & 1.677 & $1.053-2.669$ & 0.029 \\
\hline FGFR1 (subset 10 vs. subsets $1-9$ ) & 1.658 & $1.024-2.686$ & 0.040 & 1.184 & $0.690-2.029$ & 0.540 \\
\hline GATA6 (subset 10 vs. subsets 1-9) & 1.908 & $1.213-3.002$ & 0.005 & 1.605 & $0.993-2.594$ & 0.054 \\
\hline HER2 (subset 10 vs. subsets 1-9) & 0.744 & $0.412-1.343$ & 0.326 & 0.923 & $0.495-1.718$ & 0.800 \\
\hline IGF2 (subset 10 vs. subsets $1-9$ ) & 2.182 & $1.385-3.437$ & 0.001 & 2.562 & $1.591-4.125$ & $<0.001$ \\
\hline MYC (subset 10 vs. subsets 1-9) & 1.113 & $0.653-1.899$ & 0.693 & 0.955 & $0.549-1.660$ & 0.870 \\
\hline SETDB1 (subset 10 vs. subsets 1-9) & 1.638 & $1.069-2.509$ & 0.023 & 1.574 & $1.021-2.429$ & 0.040 \\
\hline
\end{tabular}

Table 1. Univariate and multivariate Cox regression analyses for overall survival and recurrence-free survival. ${ }^{a}$ Cox proportional hazards regression model, adjusted for tumor subsite, Lauren histology, lymphatic emboli, venous invasion, perineural invasion, $\mathrm{T}$ category, $\mathrm{N}$ category, and $\mathrm{M}$ category.

Gene ratios and clinicopathological features. Table 3 summarizes the relationships between the sum scores and clinicopathological features. The sum score was higher in tumors with venous invasion than in tumors without venous invasion. The sum score tended to be higher in tumors with N3b than in tumors without nodal metastasis. However, no differences in the sum score were found in association with age, sex, tumor subsite, Lauren histology, lymphatic emboli, perineural invasion, tumor depth, distant metastasis, or molecular subtype. When TIL density was compared among GCs with different sum scores, CD3 TIL and CD8 TIL densities were highest in tumors with sum scores of 0 and lowest in tumors with sum scores of 3-4 (Fig. 3a,b). When the methylation levels of repetitive DNA elements, including L1 and SAT-alpha, were compared among the three subsets, the L1 or SAT-alpha methylation level was higher in the subset with a sum score of 0 than in the subsets with a sum score of 1-2 or a sum score of 3-4 (Fig. 3c,d). However, because EBV and MSI subtypes were not classified into the copy number gain type, copy number gain status needs to be analyzed for correlation with TIL densities and L1 or SAT-alpha methylation level in non-MSI/non-EBV subtype. Not only CD3 TIL and CD8 TIL densities but also L1 or SAT-alpha methylation levels were highest in GCs with a sum score of 0 and lowest in GCs with a sum score of 3-4 (Supplementary Tables 5).

\section{Discussion}

In the present study, we analyzed the gene ratios of 7 genes, including $M Y C, E G F R, E R B B 2, F G F R 1$, GATA6, $I G F 2$, and SETDB1, in advanced GC patients using ddPCR. To determine the cut-off value of the gene ratios with prognostic utility, we partitioned the GC patients into 10 subsets according to the gene ratios and then performed survival analysis, which revealed that subset 10 with the highest gene ratios for EGFR, FGFR1, GATA6, IGF2, and SETDB1 was associated with worse clinical outcomes in patients with GC. Of these five genes, FGFR1 was not found to be an independent prognostic parameter in multivariate analysis. To assess the additive effect of copy number gains in the four genes (EGFR, GATA6, IGF2, and SETDB1), we calculated the sum score; in other words, we counted, in each case, the number of genes for which the gene ratio belonged to subset 10 . According to survival curves, the GC cases could be grouped into GCs with a sum score of 0 , a sum score of 1 or 2 , and a sum score of 3 or 4 . GCs with sum scores of 3 or 4 were found to be associated with worse survival in GC patients (OS, hazard ratio of $3.320,95 \% \mathrm{CI}=1.756-6.278, P<0.001$; RFS, hazard ratio of $3.285,95 \% \mathrm{CI}=1.736-6.217$, $P<0.001)$ in the multivariate analysis, regardless of tumor subsite, Lauren histology, venous invasion, lymphatic invasion, perineural invasion, and $\mathrm{T}, \mathrm{N}$, and $\mathrm{M}$ categories.

Our study demonstrated that the sum score was inversely associated with the CD3 or CD8 TIL density, which indicates that the copy number gain of multiple protooncogenes is associated with decreased infiltration of CD3 or CD8 TIL density. Our finding is in line with findings of recent studies in which the amplification of MYC, NOTCH2, and FGFR1 was inversely associated with the expression of genes related to cytotoxic T cell function in pancreatic ductal adenocarcinoma ${ }^{7,8}$. Not only the amplification but also the SNV mutations of protooncogenes have been demonstrated to be associated with decreased cytotoxic $\mathrm{T}$ cell infiltration in tumor areas. For lung cancers, EGFR mutations have been linked with decreased cytotoxic T cell infiltration ${ }^{9,10}$, whereas for colorectal cancers, KRAS mutations have been associated with increased marrow-derived suppressor cell infiltration and the subsequent decreased infiltration of cytotoxic T cells ${ }^{11,12}$. Based on the association between the copy number gain of multiple protooncogenes and the decreased infiltration of CD3 and CD8 TILs, it might be questioned 
(a)
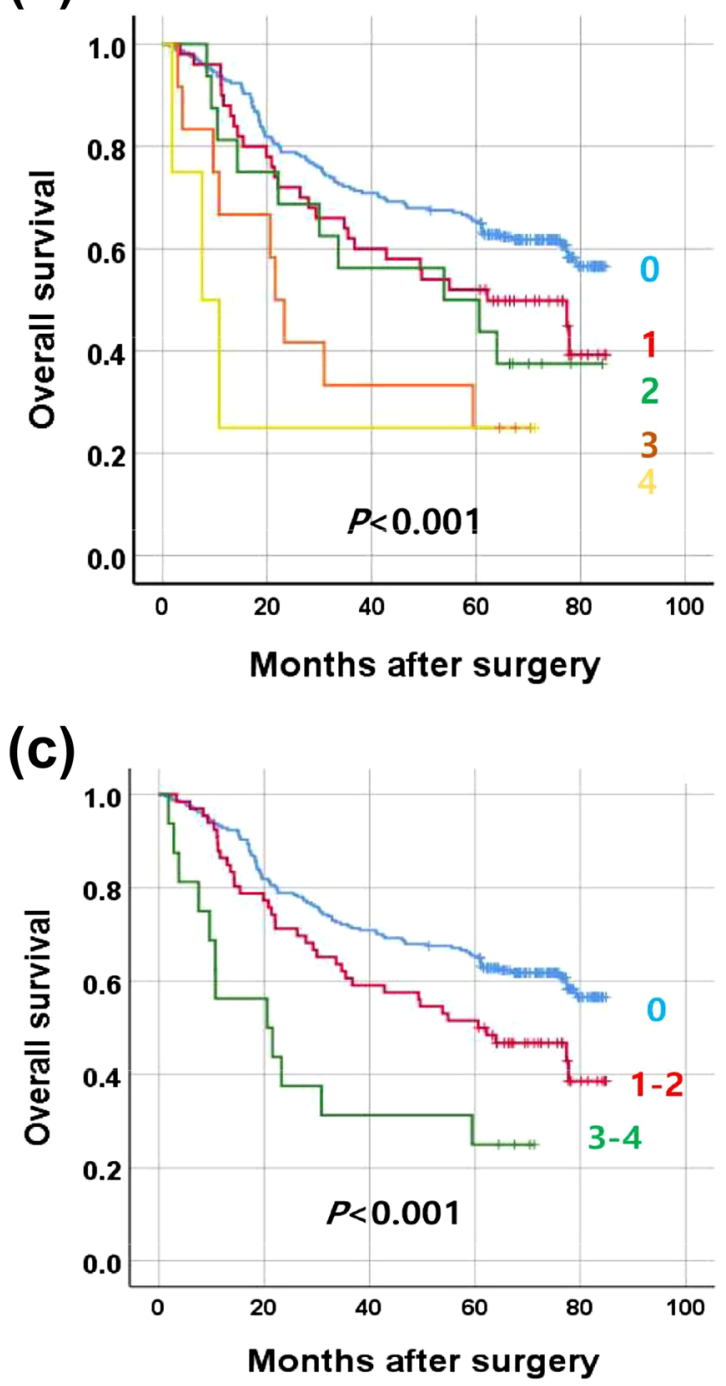

(b)

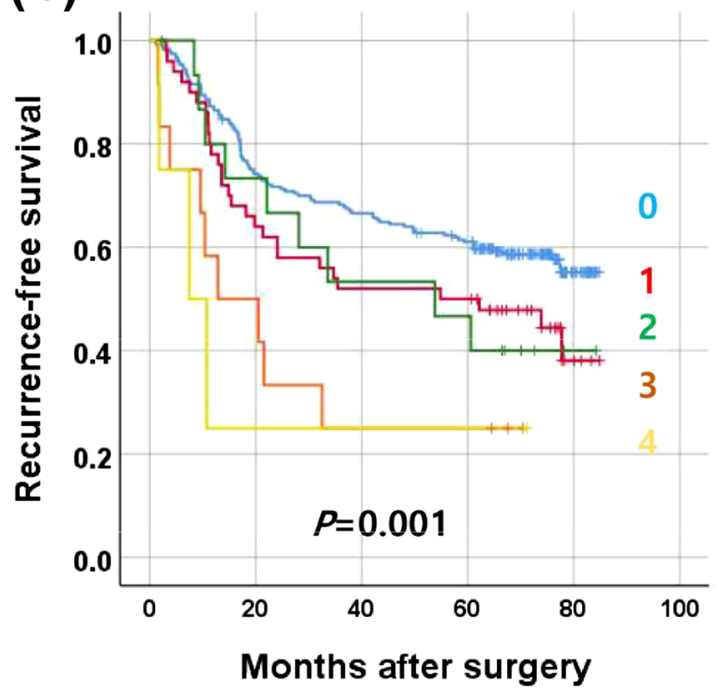

(d)

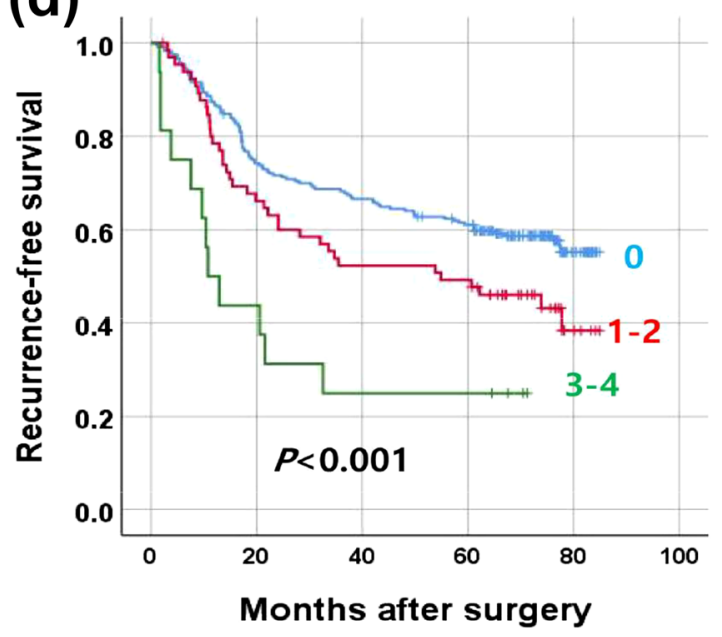

Figure 1. Survival curves for gastric cancer patients according to the sum score from 0 to $4(\mathbf{a}, \mathbf{b})$ and three subsets of gastric cancer patients according to the sum score, $0,1-2$, or 3-4 (c, d). (a, c) overall survival and (b, d) recurrence-free survival.

\begin{tabular}{|c|c|c|c|c|}
\hline & \multicolumn{2}{|l|}{ Univariate analysis } & \multicolumn{2}{|l|}{ Multivariate analysis $^{\mathrm{a}}$} \\
\hline & HR $(95 \%$ CI $)$ & $P$-value & HR $(95 \% \mathrm{CI})$ & $P$-value \\
\hline \multicolumn{5}{|c|}{ Overall survival } \\
\hline Sum score & & $<0.001$ & & $<0.001$ \\
\hline $0(\mathrm{n}=237)$ & Ref & & Ref & \\
\hline $1,2(\mathrm{n}=66)$ & $1.584(1.082-2.317)$ & 0.018 & $1.522(1.022-2.266)$ & 0.039 \\
\hline $3,4(\mathrm{n}=16)$ & $3.402(1.861-6.222)$ & $<0.001$ & $3.805(2.014-7.188)$ & $<0.001$ \\
\hline \multicolumn{5}{|c|}{ Recurrence-free survival } \\
\hline Sum score & & $<0.001$ & & $<0.001$ \\
\hline $0(\mathrm{n}=237)$ & Ref & & Ref & \\
\hline $1,2(n=66)$ & $1.512(1.037-2.205)$ & 0.032 & $1.329(0.893-1.978)$ & 0.160 \\
\hline $3,4(\mathrm{n}=16)$ & $3.235(1.773-5.902)$ & $<0.001$ & $3.709(1.953-7.042)$ & $<0.001$ \\
\hline
\end{tabular}

Table 2. Univariate and multivariate Cox regression analyses for overall survival and recurrence-free survival. ${ }^{a}$ Cox proportional hazards regression model, adjusted for tumor subsite, Lauren histology, lymphatic emboli, venous invasion, perineural invasion, T category, $\mathrm{N}$ category, and $\mathrm{M}$ category (Supplementary Tables 4 \& 5). 
(a)

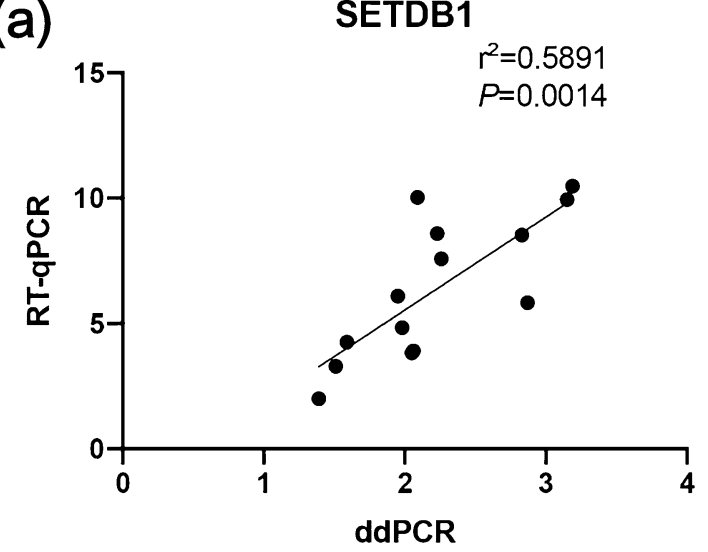

(c)

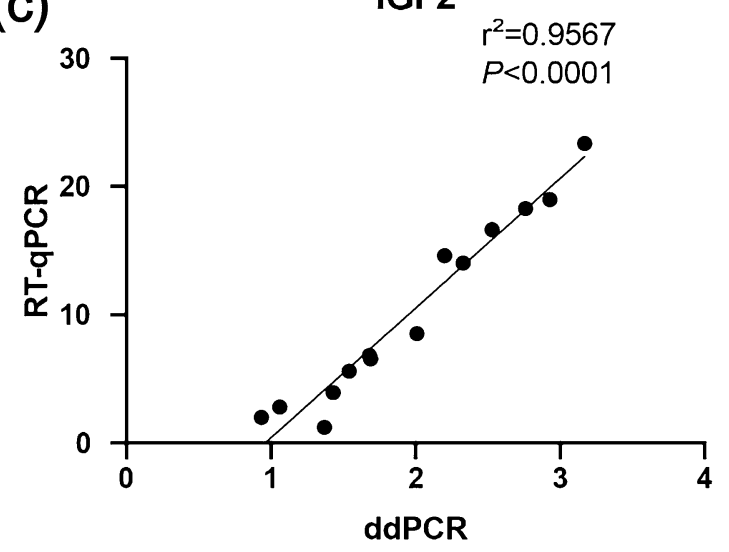

(b)

EGFR

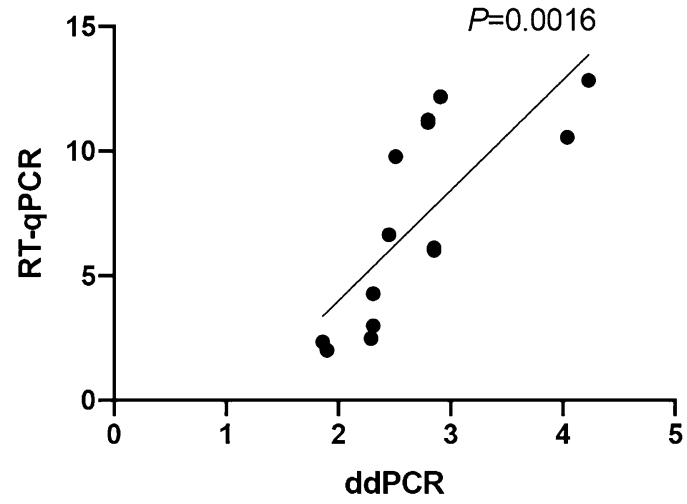

(d)

GATA6

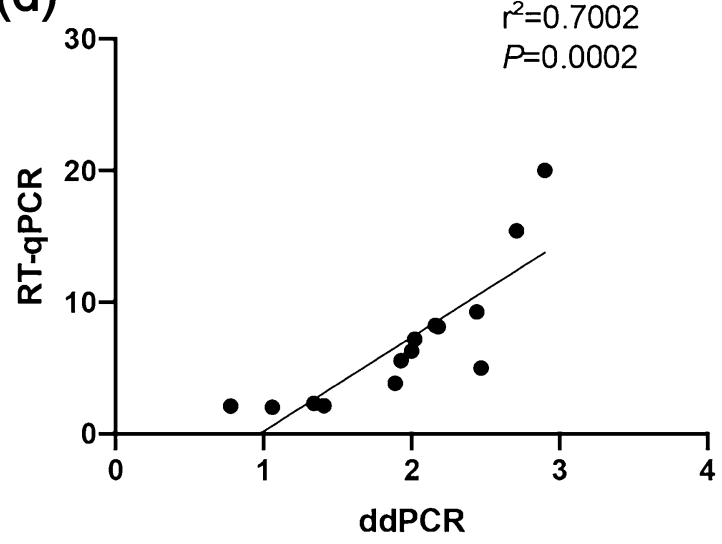

Figure 2. Correlation between RT-qPCR and ddPCR measurements. mRNA expression levels of SETDB1 (a), EGFR (b), IGF2 (c), and GATA6 (d) were measured in triplicate in 14 gastric cancer cell lines. For RT-qPCR, each dot represents the mean of the technical triplicates.

whether the prognostic value of the sum score is bestowed by the decreased density of TILs. However, in the multivariate analysis, both the sum score and CD8 TILs were found to be independent prognostic parameters for both OS and RFS (Supplementary Tables 6 \& 7).

When we correlated the sum scores with clinicopathological features, GCs with high sum scores showed an association with venous invasion but did not show associations with lymphatic emboli and nodal stage. At present, the reason why GCs with high sum scores are more likely to invade veins rather than lymphatic vessels is unclear. Whether GC cells intravasate into either blood or lymphatic vessels might be related to several factors, including the physical differences between lymphatic and blood vessels, the more favorable conditions for tumor cell survival in lymphatic vessels because of the low-shear system of fluid transport ${ }^{13}$, and the active molecular mechanisms attracting malignant cells more towards blood or lymphatic vessels ${ }^{13,14}$. In the present study, when we correlated the copy number gain of the four individual genes with venous invasion, we found that the SETDB1 gene ratio was significantly higher in GCs with venous invasion than in GCs with no venous invasion (Supplementary Table 8). The SETDB1 (KMT1E) gene encodes a histone methyltransferase that methylates Lys-9 of histone $\mathrm{H} 3$ up to trimethylation. The SETDB1 gene is located on chromosome 1q21, which shows copy number gains in several tissue types of human cancers, including breast cancer ${ }^{15}$, melanoma ${ }^{16}$, lung cancer ${ }^{17,18}$, and liver cancer ${ }^{19}$. An oncogenic role of SETDB1 has been demonstrated in lung cancer and prostate cancer, in which SETDB1 is involved in the positive stimulation of WNT signaling ${ }^{20,21}$. The downregulation of the SETDB1 gene has been found to decrease the migration and invasion of prostate cancer cells and inhibit the growth of prostate cancer cells by inducing G0/G1 cell cycle arrest ${ }^{22}$. Significant relationships between higher SETDB1 protein expression and shorter survival times have been demonstrated in patients with lung cancer ${ }^{17,23}$, liver cancer ${ }^{19}$, and colon cancer ${ }^{24}$. Although the copy number gain in GC can be referred to in the COSMIC and TCGA databases, little information is available in the literature regarding relationships between the higher expression of SETDB1 protein or the copy-number gain of SETDB1 and the clinicopathological features of GC.

Tumoral L1 hypomethylation and SAT-alpha hypomethylation have been shown to be associated with shortened survival in patients with advanced $\mathrm{GC}^{4}$. Tumoral L1 and SAT-alpha hypomethylation occurs in the background of diffuse genomic hypomethylation, which is closely associated with chromosomal instability. Thus, the copy number gain of multiple genes is expected to occur in GCs with L1 hypomethylation or SAT-alpha hypomethylation. In a previous study, we determined L1 and SAT-alpha methylation statuses using pyrosequencing methylation assays, so we used the previous data of L1 and SAT-alpha methylation levels and compared L1 


\begin{tabular}{|c|c|c|c|c|c|}
\hline & & \multicolumn{3}{|l|}{ Sum scores } & \multirow[b]{2}{*}{$P$-value } \\
\hline & & 0 & $1-2$ & $3-4$ & \\
\hline Age & & & & & 0.115 \\
\hline$<62$ years & 167 & $125(51.0 \%)$ & $38(22.8 \%)$ & $4(25.0 \%)$ & \\
\hline$\geq 62$ years & 166 & $120(49.0 \%)$ & $34(47.2 \%)$ & $12(75.0 \%)$ & \\
\hline Sex & & & & & 0.430 \\
\hline $\mathrm{M}$ & 223 & $161(65.7 \%)$ & $49(68.1 \%)$ & $13(81.3 \%)$ & \\
\hline $\mathrm{F}$ & 110 & $84(34.3 \%)$ & $23(31.9 \%)$ & $3(18.8 \%)$ & \\
\hline Site & & & & & 0.191 \\
\hline Not involving & 241 & $183(74.7 \%)$ & $46(63.9 \%)$ & $12(75.0 \%)$ & \\
\hline Involving cardia & 92 & $62(25.3 \%)$ & $26(36.1 \%)$ & $4(25.0 \%)$ & \\
\hline Lauren & & & & & 0.111 \\
\hline Intestinal & 125 & $82(33.5 \%)$ & $35(48.6 \%)$ & $8(50.0 \%)$ & \\
\hline Diffuse & 151 & $129(52.7 \%)$ & $27(37.5 \%)$ & $5(31.3 \%)$ & \\
\hline Mixed & 43 & $32(13.1 \%)$ & $8(11.1 \%)$ & $3(18.8 \%)$ & \\
\hline Unclassified & 4 & $2(0.8 \%)$ & $2(2.8 \%)$ & 0 & \\
\hline Lymphatic emboli & & & & & 0.114 \\
\hline Absent & 105 & $85(34.7 \%)$ & $16(22.2 \%)$ & $4(25.0 \%)$ & \\
\hline Present & 228 & $160(65.3 \%)$ & $56(77.8 \%)$ & $12(75.0 \%)$ & \\
\hline Venous invasion & & & & & 0.005 \\
\hline Absent & 239 & $186(75.9 \%)$ & $46(63.9 \%)$ & $7(43.8 \%)$ & \\
\hline Present & 94 & $59(24.1 \%)$ & $26(36.1 \%)$ & $9(56.3 \%)$ & \\
\hline \multicolumn{6}{|l|}{ Perineural invasion } \\
\hline Absent & 138 & $101(41.2 \%)$ & $31(43.1 \%)$ & $6(37.5 \%)$ & 0.912 \\
\hline Present & 195 & $144(58.8 \%)$ & $41(56.9 \%)$ & $10(62.5 \%)$ & \\
\hline$N$ category & & & & & 0.058 \\
\hline No & 91 & $76(31.0 \%)$ & $12(16.7 \%)$ & $3(18.8 \%)$ & \\
\hline N1 & 56 & $38(15.5 \%)$ & $15(20.8 \%)$ & $3(18.8 \%)$ & \\
\hline N2 & 68 & $50(20.4 \%)$ & $17(23.6 \%)$ & $1(6.3 \%)$ & \\
\hline N3a & 72 & $52(21.2 \%)$ & $17(23.6 \%)$ & $3(18.8 \%)$ & \\
\hline $\mathrm{N} 3 \mathrm{~b}$ & 46 & $29(11.8 \%)$ & $11(15.3 \%)$ & $6(37.5 \%)$ & \\
\hline T category & & & & & 0.336 \\
\hline $\mathrm{T} 2$ & 61 & $51(20.8 \%)$ & $10(13.9 \%)$ & 0 & \\
\hline T3 & 121 & $90(36.7 \%)$ & $24(33.3 \%)$ & $7(43.8 \%)$ & \\
\hline $\mathrm{T} 4 \mathrm{a}$ & 134 & $91(37.1 \%)$ & $34(47.2 \%)$ & $8(50.0 \%)$ & \\
\hline $\mathrm{T} 4 \mathrm{~b}$ & 18 & $13(5.3 \%)$ & $4(5.6 \%)$ & $1(6.3 \%)$ & \\
\hline \multicolumn{6}{|l|}{$M$ category } \\
\hline M0 & 284 & $211(86.1 \%)$ & $57(79.2 \%)$ & $16(100.0 \%)$ & 0.080 \\
\hline M1 & 49 & $34(13.9 \%)$ & $15(20.8 \%)$ & 0 & \\
\hline Molecular subtype & & & & & 0.161 \\
\hline MSS/EBV- & 265 & $189(77.1 \%)$ & $60(83.3 \%)$ & $16(100.0 \%)$ & \\
\hline MSI-H & 42 & $33(13.5 \%)$ & $9(12.5 \%)$ & 0 & \\
\hline EBV+ & 26 & $23(9.4 \%)$ & $3(4.2 \%)$ & 0 & \\
\hline
\end{tabular}

Table 3. Comparison of clinicopathological features according to the sum scores. Abbreviations: MSS, microsatellite-stable; EBV-, EBV-negative; MSI-H, high level of microsatellite instability; EBV+, EBV-positive.

and SAT-alpha methylation levels among different sum scores, which revealed a significant difference between GCs with sum scores of 0 and GCs with sum scores of 1-2 or sum scores of 3-4 (Fig. 3). To identify whether the prognostic significance of the sum score could be affected by L1 and SAT-alpha methylation statuses, we performed multivariate analysis with the inclusion of L1 and SAT-alpha methylation statuses and other prognostic variables that were found to be statistically significant in the univariate survival analysis (Supplementary Table 9). The sum score was found to be an independent prognostic parameter for both OS and RFS.

There are a few limitation to the current study. One of which is a lack of validation set. As an attempt to overcome this shortcoming, we applied our scoring system on identical genes of TCGA STAD. However, only a small fraction of samples scored higher than $3(4 / 438,0.9 \%)$ in TCGA STAD compared with our study cohort $(16 / 319,5.0 \%)$ and such as small sample size will not suffice as proper validation. Therefore, an independent, external validation set would be essential in future studies. 

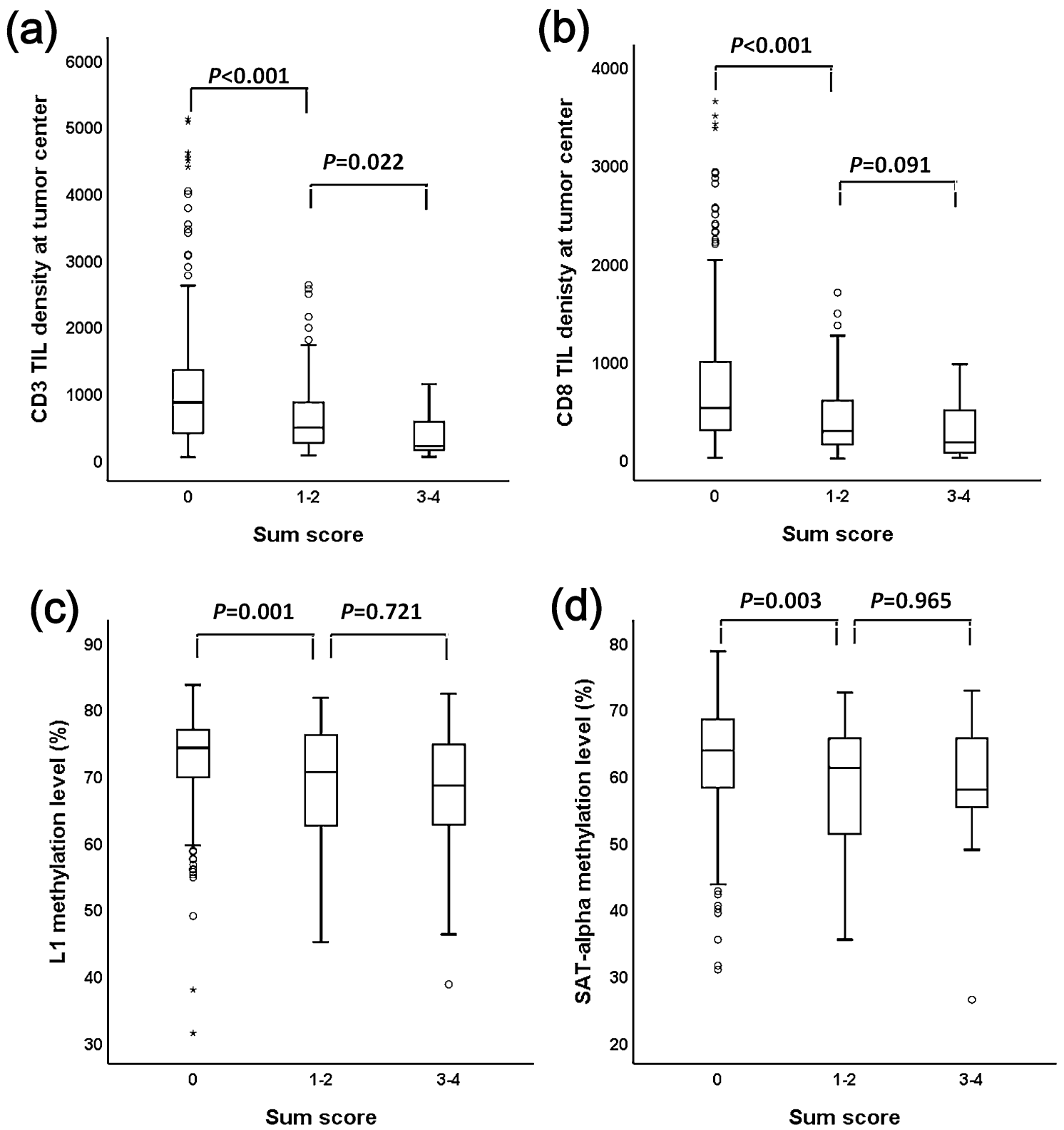

Figure 3. Density of tumor-infiltrating lymphocytes and methylation levels of repetitive DNA elements in gastric carcinomas according to sum score. $(\mathbf{a}, \mathbf{b}) \mathrm{CD} 3$ and CD8 tumor-infiltrating lymphocyte densities and (c, d) L1 and SAT-alpha methylaiton densities.

In conclusion, copy number gains in three or four of the EGFR, GATA6, IGF2, and SETDB1 genes were found to be associated with venous invasion, decreased TIL densities, decreased levels of DNA methylation in L1 or SAT-alpha, and shortened rates of both OS and RFS. A high sum score was found to be an independent prognostic parameter associated with poor prognosis in patients with advanced GC. An independent study is needed to validate the prognostic value of high sum scores in the four genes.

\section{Data availability}

All Relevant data are within the paper.

Received: 27 January 2021; Accepted: 28 May 2021

Published online: 09 June 2021

\section{References}

1. Sohn, B. H. et al. Clinical significance of four molecular subtypes of gastric cancer identified by the cancer genome atlas project. Clin. Cancer Res. https://doi.org/10.1158/1078-0432.CCR-16-2211 (2017).

2. Cancer Genome Atlas Research, N. Comprehensive molecular characterization of gastric adenocarcinoma. Nature 513, 202-209. https://doi.org/10.1038/nature13480 (2014). 
3. Song, Y. S. et al. Methylation status of long interspersed element-1 in advanced gastric cancer and its prognostic implication. Gastric Cancer 19, 98-106. https://doi.org/10.1007/s10120-015-0463-6 (2016).

4. Kim, Y. et al. Combinatory low methylation statuses of SAT-alpha and L1 are associated with shortened survival time in patients with advanced gastric cancer. Gastric Cancer 22, 37-47. https://doi.org/10.1007/s10120-018-0852-8 (2019).

5. Kim, Y. et al. Combination of L1 methylation and tumor-infiltrating lymphocytes as prognostic marker in advanced gastric cancer. Gastric Cancer https://doi.org/10.1007/s10120-019-01025-8 (2019).

6. Whale, A. S. et al. Comparison of microfluidic digital PCR and conventional quantitative PCR for measuring copy number variation. Nucleic Acids Res. 40, https://doi.org/10.1093/nar/gks203 (2012).

7. Bailey, P. et al. Genomic analyses identify molecular subtypes of pancreatic cancer. Nature 531, 47-52. https://doi.org/10.1038/ nature16965 (2016).

8. Balli, D., Rech, A. J., Stanger, B. Z. \& Vonderheide, R. H. Immune cytolytic activity stratifies molecular subsets of human pancreatic cancer. Clin. Cancer Res. 23, 3129-3138. https://doi.org/10.1158/1078-0432.CCR-16-2128 (2017).

9. Concha-Benavente, F. \& Ferris, R. L. Reversing EGFR mediated immunoescape by targeted monoclonal antibody therapy. Front Pharmacol. 8, 332. https://doi.org/10.3389/fphar.2017.00332 (2017).

10. Busch, S. E. et al. Lung cancer subtypes generate unique immune responses. J. Immunol. 197, 4493-4503. https://doi.org/10.4049/ jimmunol.1600576 (2016).

11. Liao, W. et al. KRAS-IRF2 axis drives immune suppression and immune therapy resistance in colorectal cancer. Cancer Cell 35, 559-572 e557. https://doi.org/10.1016/j.ccell.2019.02.008 (2019).

12. Lal, N. et al. KRAS mutation and consensus molecular subtypes 2 and 3 are independently associated with reduced immune infiltration and reactivity in colorectal cancer. Clin. Cancer Res. 24, 224-233. https://doi.org/10.1158/1078-0432.CCR-17-1090 (2018).

13. Wong, S. Y. \& Hynes, R. O. Lymphatic or hematogenous dissemination: How does a metastatic tumor cell decide?. Cell Cycle 5, 812-817. https://doi.org/10.4161/cc.5.8.2646 (2006).

14. Aleskandarany, M. A., Sonbul, S. N., Mukherjee, A. \& Rakha, E. A. Molecular mechanisms underlying lymphovascular invasion in invasive breast cancer. Pathobiology 82, 113-123. https://doi.org/10.1159/000433583 (2015).

15. Liu, L., Kimball, S., Liu, H., Holowatyj, A. \& Yang, Z. Q. Genetic alterations of histone lysine methyltransferases and their significance in breast cancer. Oncotarget 6, 2466-2482. https://doi.org/10.18632/oncotarget.2967 (2015).

16. Ceol, C. J. et al. The histone methyltransferase SETDB1 is recurrently amplified in melanoma and accelerates its onset. Nature 471, 513-517. https://doi.org/10.1038/nature09806 (2011).

17. Inoue, Y. et al. Clinicopathological and survival analysis of japanese patients with resected non-small-cell lung cancer harboring NKX2-1, SETDB1, MET, HER2, SOX2, FGFR1, or PIK3CA gene amplification. J. Thorac. Oncol. 10, 1590-1600. https://doi.org/ 10.1097/JTO.0000000000000685 (2015).

18. Rodriguez-Paredes, M. et al. Gene amplification of the histone methyltransferase SETDB1 contributes to human lung tumorigenesis. Oncogene 33, 2807-2813. https://doi.org/10.1038/onc.2013.239 (2014).

19. Wong, C. M. et al. Up-regulation of histone methyltransferase SETDB1 by multiple mechanisms in hepatocellular carcinoma promotes cancer metastasis. Hepatology 63, 474-487. https://doi.org/10.1002/hep.28304 (2016).

20. Sun, Q. Y. et al. SETDB1 accelerates tumourigenesis by regulating the WNT signalling pathway. J. Pathol. 235, 559-570. https:// doi.org/10.1002/path.4482 (2015).

21. Li, Q. et al. Quantitative proteomic study of human prostate cancer cells with different metastatic potentials. Int. J. Oncol. 48, 1437-1446. https://doi.org/10.3892/ijo.2016.3378 (2016).

22. Sun, Y. et al. Histone methyltransferase SETDB1 is required for prostate cancer cell proliferation, migration and invasion. Asian J. Androl. 16, 319-324. https://doi.org/10.4103/1008-682X.122812 (2014).

23. Lafuente-Sanchis, A. et al. Prognostic value of ERCC1, RRM1, BRCA1 and SETDB1 in early stage of non-small cell lung cancer. Clin. Transl. Oncol. 18, 798-804. https://doi.org/10.1007/s12094-015-1440-6 (2016).

24. Ho, Y. J. et al. Significance of histone methyltransferase SETDB1 expression in colon adenocarcinoma. APMIS 125, 985-995. https://doi.org/10.1111/apm.12745 (2017).

\section{Acknowledgements}

This work was financially supported by a grant from the National Research Foundation (NRF) funded by the Korean Ministry of Science and ICT (2019R1F1A1061227) and a grant from the Korea Health Technology R\&D Project through the Korea Health Industry Development Institute (KHIDI), funded by the Ministry of Health \& Welfare, Republic of Korea (grant number: HI14C1277).

\section{Author contributions}

Designing the study: G.H.K. Preparing the manuscript: M.L. and Y.H.K. Concept of study: G.H.K. and T.K. Prepare material and data: Y.H.K., N.Y.C., J.M.B. and W.H.K. Experiment: M.L., T.K. and N.Y.C. Edit manuscript: G.H.K. and Y.H.K. Prepare figures: M.L. and G.H.K. Data analysis: M.L., Y.K. and G.H.K. Statistics: G.H.K. and Y.K.

\section{Competing interests}

The authors declare no competing interests.

\section{Additional information}

Supplementary Information The online version contains supplementary material available at https:/doi.org/ 10.1038/s41598-021-91652-y.

Correspondence and requests for materials should be addressed to G.H.K.

Reprints and permissions information is available at www.nature.com/reprints.

Publisher's note Springer Nature remains neutral with regard to jurisdictional claims in published maps and institutional affiliations. 
(c) (i) Open Access This article is licensed under a Creative Commons Attribution 4.0 International cc) License, which permits use, sharing, adaptation, distribution and reproduction in any medium or format, as long as you give appropriate credit to the original author(s) and the source, provide a link to the Creative Commons licence, and indicate if changes were made. The images or other third party material in this article are included in the article's Creative Commons licence, unless indicated otherwise in a credit line to the material. If material is not included in the article's Creative Commons licence and your intended use is not permitted by statutory regulation or exceeds the permitted use, you will need to obtain permission directly from the copyright holder. To view a copy of this licence, visit http://creativecommons.org/licenses/by/4.0/.

(C) The Author(s) 2021 